\title{
Raman scattering studies of ultrashallow Sb implants in strained Si
}

\author{
L. O'Reilly $\cdot$ N. S. Bennett $\cdot$ P. J. McNally $\cdot$ \\ B. J. Sealy · N. E. B. Cowern · A. Lankinen • \\ T. O. Tuomi
}

Published online: 21 June 2007

(C) Springer Science+Business Media, LLC 2007

\begin{abstract}
Sheet resistance $\left(R_{\mathrm{S}}\right)$ reductions are presented for antimony doped layers in strained Si. We use microRaman spectroscopy to characterise the impact of a low energy ( $2 \mathrm{keV}$ ) Sb implantation into a thin strained Si layer on the crystalline quality and resultant stress in the strained $\mathrm{Si}$. The use of $325 \mathrm{~nm}$ UV laser light enables us to extract information from the top $\sim 9 \mathrm{~nm}$ of the strained Si layer. Prior to implantation the Si layer is fully strained with a tensile stress value $\sim 1.41 \mathrm{GPa}$, in agreement with the calculated theoretical maximum on a strain relaxed buffer with $17 \%$ Ge content. There is a clear decrease in the intensity of the Si Raman signal following Sb implantation. The lattice damage and lattice recovery achieved by subsequent rapid thermal anneal (RTA) is quantified using the amplitude and full width at half maximum (FWHM) of the crystalline Si peak. The shift of the Raman Si peak is a key parameter in the interpretation of the spectra. The ion-implanted sample is studied in terms of a phonon coherence length confinement model. Carrier concentration effects are seen to play a role in the Raman shift following electrical activation of the $\mathrm{Sb}$ atoms by RTA.
\end{abstract}

L. O'Reilly $(\bowtie) \cdot$ P. J. McNally

Nanomaterials Processing Laboratory,

Research Institute for Networks and Communications

Engineering (RINCE), School of Electronic Engineering,

Dublin City University, Glasnevin, Dublin 9, Ireland

e-mail: oreillyl@eeng.dcu.ie

N. S. Bennett · B. J. Sealy · N. E. B. Cowern

Advanced Technology Institute, University of Surrey, Guildford GU2 7XH, UK

A. Lankinen - T. O. Tuomi

Micro and Nanosciences, Micronova, Helsinki University of Technology, P.O. Box 3500, Espoo 02015 TKK, Finland

\section{Introduction}

The ability to improve performance consistently while decreasing power consumption has made CMOS architecture the dominant technology for integrated circuits. The scaling of the CMOS transistor has been the primary factor driving improvements in microprocessor performance [1]. Strain engineering using $\mathrm{Si} / \mathrm{SiGe}$ heterostructures is a key technology for the enhancement of device operating speeds. The production of ultrashallow junctions for the source/drain extension region using low energy ion-implantation will be required for future CMOS devices. The extent to which strain engineering can be successfully combined with ionimplantation doping of ultrashallow source-drain regions will depend on the effects of strain on dopant diffusion and activation at high dopant concentrations. Arsenic is the standard n-type dopant species in Si. We investigate $\mathrm{Sb}$ as a possible alternative to As, due to improvements in junction depth and abruptness resulting from its greater mass.

\section{Experimental details}

Experiments were performed on IQE tensile strained $\mathrm{Si}$ wafers grown on constant-composition $\mathrm{Si}_{0.83} \mathrm{Ge}_{0.17}$ relaxed buffer layers. Each wafer was implanted with a low energy (2 keV) Sb implant to a dose of $4 \times 10^{14} \mathrm{~cm}^{-2}$ creating a junction at $12 \mathrm{~nm}$, as defined by dopant concentration falloff to the $3 \times 10^{18} \mathrm{~cm}^{-3}$ level. Control samples were prepared using conventional p-type Si wafers for comparison. The quality of the SiGe substrates was investigated using white beam synchrotron X-ray topography. The synchrotron X-ray topography measurements were performed at HASYLAB-DESY and ANKA. The Hamburger Synchrotronstrahlungslabor am Deutschen Elektronen-Synchrotron 
(HASYLAB-DESY) utilises the continuous spectrum of synchrotron radiation from the DORIS storage ring bending magnet. The ring operates at positron energies of $4.45 \mathrm{GeV}$ and at typical currents of $80-150 \mathrm{~mA}$. At the recently constructed Angströmquelle Karlsruhe, (ANKA, Karlsruhe, Germany) synchrotron light source, a small point source of $0.5 \times 0.2 \mathrm{~mm}^{2}$ at a bending magnet of the $2.5 \mathrm{GeV}$ storage ring provides light with a characteristic wavelength of $2 \AA$ and typical beam currents of $100-$ $200 \mathrm{~mA}$. The topograph patterns are recorded on highresolution Slavich VRP-M X-ray films with a grain size of approximately $0.05 \mu \mathrm{m}$. Van der Pauw sheet resistance measurements were used to compare the resistivity of Sb-implanted samples in unstrained and strained Si wafers to values obtained for As in strained Si. Room temperature micro-Raman measurements were performed with a Jobin Yvon LabRam HR800 system in backscattering geometry using a $325 \mathrm{~nm} \mathrm{He}-\mathrm{Cd}$ UV laser excitation with a spot size of approximately $1 \mu \mathrm{m}$. Each spectrum was averaged over at least ten acquisitions to reduce noise effects.

\section{Results and discussion}

\subsection{Synchrotron X-ray topography}

An example of a large area, back-reflection topograph is shown in Fig. 1. The penetration depth $\left(t_{\mathrm{p}}\right)$ of the X-rays for this $1 \overline{1} 7$ reflection is $15.8 \mu \mathrm{m}$. Therefore the entire SiGe buffer layer is being imaged in this geometry. The projection of the diffraction vector $g$, onto the plane of the recording film is indicated in the figure. We see a welldefined crosshatched pattern with ridges running along two

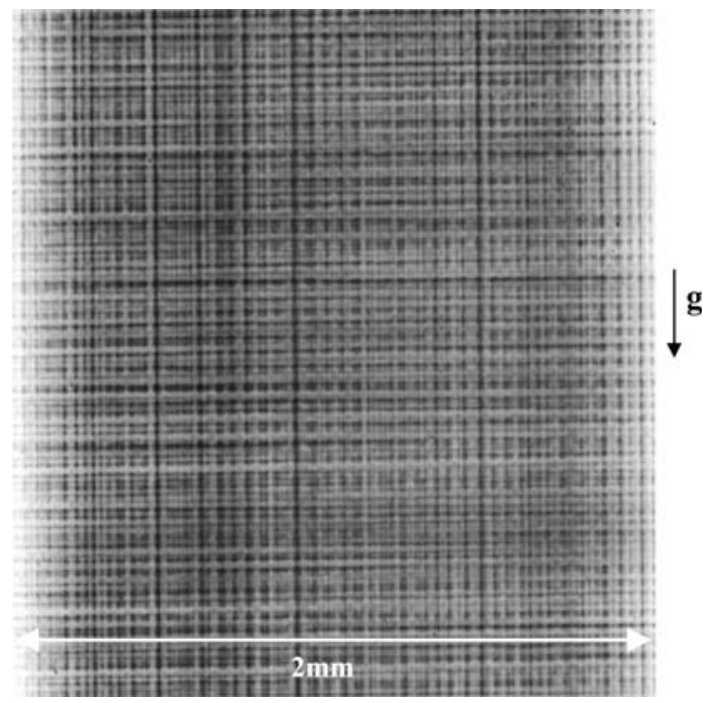

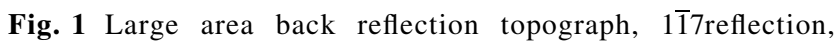
$t_{\mathrm{p}}=15.8 \mu \mathrm{m}$ perpendicular $\langle 011\rangle$ directions. This is characteristic of misfit dislocations. The presence of misfit dislocations verifies that there is strain relaxation in the $\mathrm{SiGe}$ buffer layers, as expected. We do not see any evidence of other defects in the topograph, confirming that the substrates used are of high quality.

\subsection{Electrical measurements}

Van der Pauw sheet resistance measurements as a function of anneal temperature are shown in Fig. 2. A significant reduction in $R_{\mathrm{s}}$ is measured for $\mathrm{Sb}$ implants in strained $\mathrm{Si}$ compared to bulk unstrained material. The reduction in electrical activation, resulting in an increased $R_{\mathrm{s}}$ value of $\mathrm{Sb}$ in bulk $\mathrm{Si}$ at $800{ }^{\circ} \mathrm{C}$, is in agreement with studies by Alzanki et al. [2]. Previous investigations of the electrical properties have indicated that this $R_{\mathrm{S}}$ lowering in strained $\mathrm{Si}$, results not only from strain enhanced mobility, but also from an improvement in electrical activation of $\mathrm{Sb}$ with strain [3]. Earlier work examining As indicates that this $\mathrm{Sb}$ behaviour differs from that of As in which sheet resistance measurements of As in bulk and strained-Si layers performed by Dilliway et al. [4] showed a reduction in the $R_{\mathrm{s}}$ value in strained material resulting from a $15 \%$ higher electron mobility in the strained $(20 \% \mathrm{Ge})$ material, but no evidence was found for a difference in electrically active As concentrations as a function of strain. This is confirmed here, by our comparison of the sheet resistance values of $\mathrm{Sb}$ and As in strained Si under identical implant and annealing conditions showing a clear decrease in $R_{\mathrm{s}}$ for the $\mathrm{Sb}$ case.

\subsection{Micro-Raman spectroscopy}

The Raman spectra of the strained-Si and reference $\mathrm{Si}$ samples (Fig. 3) are dominated by the signal of the

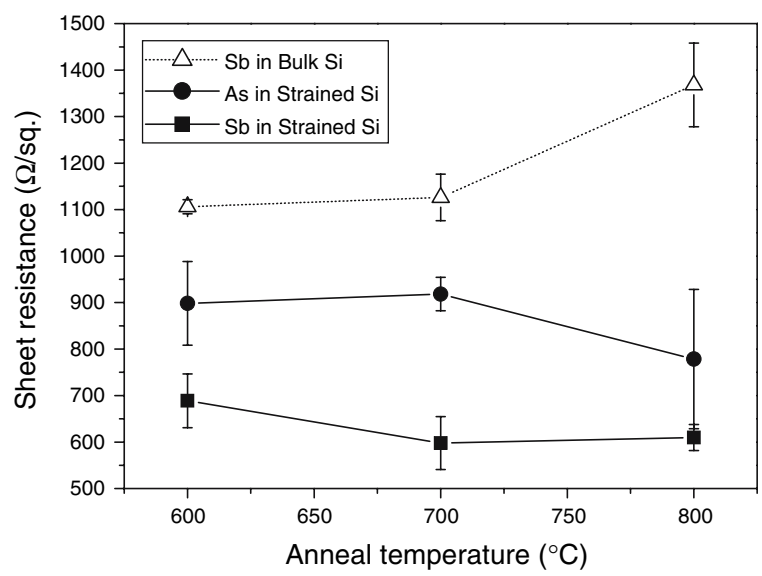

Fig. $2 R_{\mathrm{S}}$ measurements as a function of annealing temperature for $2 \mathrm{keV}, 4 \times 10^{14} \mathrm{~cm}^{-2} \mathrm{Sb}$ implants into bulk and strained $\mathrm{Si}$, and $\mathrm{As}$ implants in strained $\mathrm{Si}$ 
degenerate Si-Si Raman peak. For the bulk Si wafer, this $\mathrm{Si}$ peak appears at a Raman shift of $\sim 520 \mathrm{~cm}^{-1}$. A red shift (lower wavenumber) in the $\mathrm{Si}-\mathrm{Si}$ Raman peak position of the strained Si samples compared to that of the reference unstrained $\mathrm{Si}$ sample confirms that a tensile stress is present in the $\mathrm{Si}$ cap layer. Using the average $\mathrm{Si}-\mathrm{Si}$ phonon peak position, the biaxial stress in the $\mathrm{Si}$ cap layer before $\mathrm{Sb}$ implantation was calculated from $\Delta \omega_{\mathrm{Si}_{\mathrm{UV}}}$ using the equation $\sigma_{\mathrm{xx}}=\sigma_{\mathrm{xy}}=-\Delta \omega_{\mathrm{Si}_{\mathrm{UV}}} / 4(\mathrm{GPa})$ [5], where $\Delta \omega_{\mathrm{Si}}$ is the $\mathrm{Si}-\mathrm{Si}$ Raman peak shift compared to that of the reference $\mathrm{Si}$ sample obtained using the UV laser, and $\sigma_{\mathrm{xx}}=\sigma_{\mathrm{yy}}$ are the stress components of the biaxial stress in the $x-y$ plane. Therefore the measured peak shift of $-5.64 \pm 0.1 \mathrm{~cm}^{-1}$ corresponds to a stress of $1.41 \pm 0.03 \mathrm{GPa}$. In the case of a fully strained Si cap layer, the tensile stress $\sigma_{\mathrm{f}}$ in the Si cap can be calculated with the following equation [6]: $\sigma_{\mathrm{f}}=Y_{\mathrm{f}} m /$ $(1-v)$, where $Y_{\mathrm{f}}$ is the film Young's modulus, $m$ is the misfit between the film and the substrate lattice parameters and $v$ is Poisson's ratio of the film. By estimating the lattice constant of a fully relaxed $\mathrm{Si}_{0.83} \mathrm{Ge}_{0.17}$ the misfit between the Si cap and SiGe buffer layer is found to be $\sim 0.71 \%$. Thus the tensile stress for a fully strained $\mathrm{Si}$ layer on $\mathrm{Si}_{0.83} \mathrm{Ge}_{0.17}$ is calculated as $1.28 \mathrm{GPa}$, which is close to the experimentally measured value.

The Si Raman peak intensity before and after implantation and heat treatment for both strained $\mathrm{Si}$ and bulk $\mathrm{Si}$ samples is plotted in Fig. 4. It is noted that before ion implantation, the Si Raman peak amplitude of the strained $\mathrm{Si}$ sample is somewhat lower than that of the bulk Si reference and the FWHM of the strained Si peak, $3.8 \mathrm{~cm}^{-1}$ is slightly larger than that of the Raman peak of the bulk Si, $3.5 \mathrm{~cm}^{-1}$. This suggests that the crystalline quality of the thin, $17 \mathrm{~nm}$ strained Si film is not quite as good as that of the bulk Si wafer. Following Sb implantation, the intensity of the $\mathrm{Si}-\mathrm{Si}$ mode Raman peak decreases to approximately

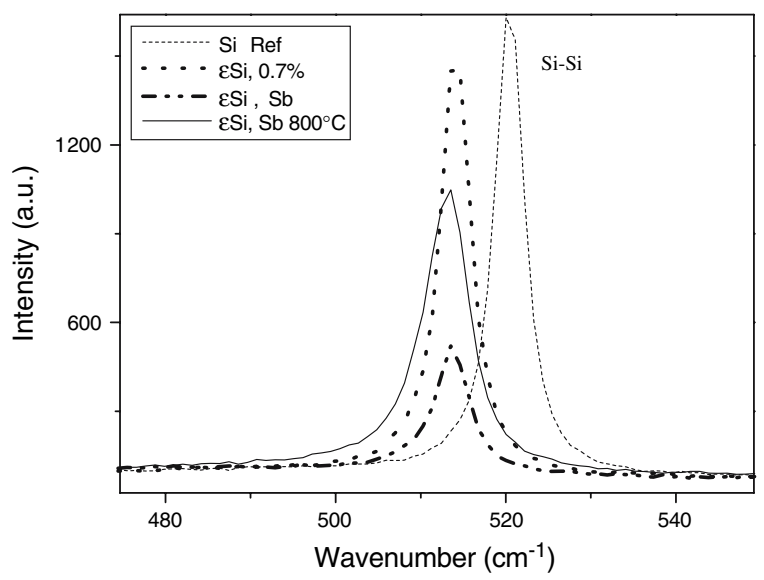

Fig. 3 UV Raman spectra of Sb-implanted and strain free $\mathrm{Si}$ reference sample

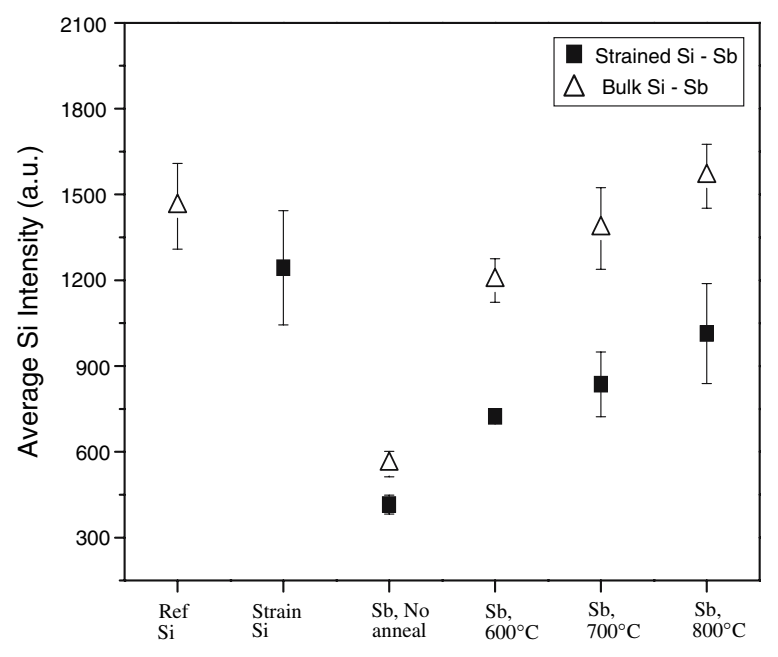

Fig. 4 Average Peak amplitude of the $\mathrm{Si}-\mathrm{Si}$ Raman mode in Sbimplanted Strained and Bulk Si substrates

half of that of the strained Si sample prior to implantation and the FWHM increases from $3.8 \mathrm{~cm}^{-1}$ to $4.3 \mathrm{~cm}^{-1}$, respectively, indicating an increase in lattice disorder. After RTA at $600{ }^{\circ} \mathrm{C}$ for $10 \mathrm{~s}$, the lattice damage induced by the ion-implantation begins to be repaired, and therefore the amplitude of the Si peak increases. A further increase in intensity is detected following RTA at 700 and $800{ }^{\circ} \mathrm{C}$. The amplitude of the Si Raman peak has recovered to its original value in the case of the bulk Si after annealing at $800{ }^{\circ} \mathrm{C}$. However, the lattice recovery is not complete in the strained Si samples.

The Si Raman peak position was extracted using a Gauss/Lorentz fitting function for each of the strained $\mathrm{Si}$ and bulk Si samples. The Raman shift relative to the reference $\mathrm{Si}$ peak position for bulk samples and the normalised Raman shift relative to the strained Si peak position for strained Si samples are compared in Fig. 5 as a function of annealing temperature. A red shift of the Raman peak is detected following Sb implantation and this increases with RTA at $600{ }^{\circ} \mathrm{C}$. A number of factors may contribute to this $\mathrm{Si}$ Raman peak shift, including stress, phonon confinement effects [7] and increased carrier concentration [8]. The broadening of the Si Raman peak and red-shift of the peak position is indicative of confinement effects resulting from partial amorphisation of the strained $\mathrm{Si}$ layer during ion implantation. Since translational symmetry breakdown is expected in the ion-implanted samples resulting in a relaxation of the momentum conservation rule, the Raman spectra were studied in terms of a phonon correlation length model. This confinement model, first developed by Richter [9] and Campbell and Fauchet [10] is modified slightly to model our Raman spectra following Sb ion-implantation. In a perfect crystalline semiconductor, the long-range 


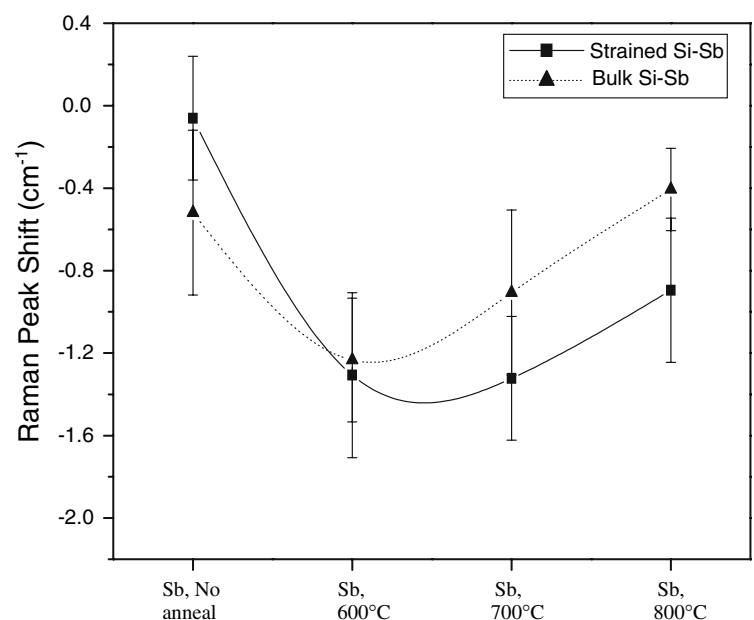

Fig. 5 Normalised peak shift of the $\mathrm{Si}-\mathrm{Si}$ Raman mode in Sbimplanted Strained and Bulk Si substrates

periodicity of the lattice makes the correlation length of the normal-mode vibrations infinite, giving rise to the momentum selection rule, which limits Raman scattering to zone-centre $(q=0)$ optical phonon modes. The bulk, plane-wave-like phonon wavefunction cannot exist within a small crystallite. The spatial confinement, via the Heisenberg uncertainty principle, results in light scattering from a nominally zone-centre phonon whose wavevector has an uncertainty $\Delta q$ and whose energy thus has an uncertainty $\Delta \omega$. Thus the phonon's spatial confinement results in a broadening of the Raman scattering features reflecting the uncertainty in its energy and a red shift of the Raman peak position occurs with increasing spatial confinement, as lower-energy bulk-like phonons are incorporated into the wavefunction describing the nominally zone-centre confined phonon. The model used by Macia et al. [7] to study silicon-on-insulator samples obtained by high dose oxygen ion implantation is adapted by introducing the parameter $\omega_{0}$, which represents the stress-induced Raman line shift. Assuming a constant correlation length $L$ in the scattering volume, the intensity of the first order Raman band of silicon is given by

$I(\omega) \propto \int_{0}^{2 \pi / a_{0}} \frac{|C(q)|^{2} 4 \pi q^{2} d q}{\left(\omega-\omega_{0}-\omega(q)\right)^{2}+\left(\Gamma_{0} / 2\right)^{2}}$

where $a_{0}$ is the lattice constant of $\mathrm{Si}$ and $\Gamma_{0}$ is the Raman intrinsic line width of crystalline Si. The weight factor $C(q)$ for the scattering with wave vector $q$ is given by

$|C(q)|^{2} \propto \exp \left(\frac{q^{2} L^{2}}{8}\right)$

$\omega(q)$ is the phonon dispersion relation, taken as $\omega(q)=\omega_{\mathrm{p}}-120\left(\frac{q}{q_{0}}\right)^{2}$

where $\omega_{\mathrm{p}}$ is the wave number of the first order Raman band in the absence of disorder effects $\left(520 \mathrm{~cm}^{-1}\right)$ and $q_{0}=2 \pi /$ $a_{0}$. The experimental data were fitted by varying the parameters $\omega_{0}$ and $L$, so that the stress effect (contained in the parameter $\omega_{0}$ ) can be decoupled from those due to residual damage (contained in the parameter $L$ which gives an indication of the average distance between defects). The result of the fitting procedure is shown in Fig. 6. The phonon correlation length estimated from the theoretical fit of the spectrum is $30 \AA$. The intrinsic Si line width was chosen as $3.5 \mathrm{~cm}^{-1}$, in agreement with our FWHM value obtained for bulk Si, and $\omega_{0}$ was set to 5.3 to account for the shift in the Raman spectrum due to stress. A shift of $5.3 \mathrm{~cm}^{-1}$ implies a tensile stress of $1.33 \mathrm{GPa}$, which indicates that slight relaxation of the thin strained Si layer (measured stress of $1.41 \mathrm{GPa}$ before implantation) has occurred during ion implantation. The small net red-shift of the Si Raman peak position of the strained Si sample following ion implantation, of the order of $-0.1 \mathrm{~cm}^{-1}$ (Fig. 5) is accounted for by confinement effects.

This confinement model is no longer valid for the annealed samples due to the recovery of the lattice. However, a further increase in the red-shift of the Si Raman peak occurs following RTA at $600{ }^{\circ} \mathrm{C}$. Due to the difficulty of extracting very small differences in the Raman Si peak position, the measurements were repeated several times and the error bars plotted in Fig. 5 represent a standard deviation from the average value. Although the error bars are not insignificant, a clear general trend emerges where the maximum Si peak shift is found in the range of 600 $700{ }^{\circ} \mathrm{C}$ in both bulk and strained $\mathrm{Si}$, and a small reduction in the red-shift is detected in samples annealed at $800{ }^{\circ} \mathrm{C}$.

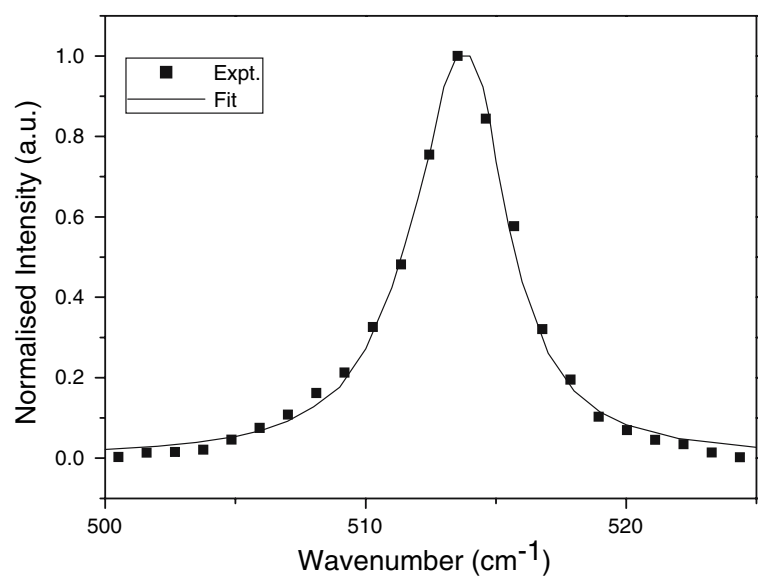

Fig. 6 Experimental points and theoretical (solid line) fit of the Raman spectrum of strained $\mathrm{Si}$ after Sb implantation 
An early study on the dependence of the first-order Raman frequency of n-type $\mathrm{Si}$ as a function of carrier concentration found that the frequency of the Si peak decreases as the electron carrier concentration increases [8]. This effect can be explained by a lowering of the average energy of the free carriers due to the splitting of the X-point degeneracy of the conduction band, resulting in a smaller contribution to the free energy, which produces a softening of the lattice [8]. Measurements of the sheet carrier concentration of these samples recently published by us [11] have shown maximum values at $600-700{ }^{\circ} \mathrm{C}$, before a reduction in carrier concentration at $800{ }^{\circ} \mathrm{C}$. This result agrees with the measured shift in the Si Raman peak position following heat treatment. For annealed samples, the absolute values of the Raman peak shift are smaller in the case of bulk $\mathrm{Si}$ compared to strained Si. Again this is consistent with the interpretation that a carrier concentration effect is playing a role in the Raman peak shift as we have shown that $0.7 \%$ strain has doubled the amount of electrically active $\mathrm{Sb}$ compared to $\mathrm{Sb}$ implants in bulk Si [11]. Cerdeira and Cardona [8] developed a formula describing the Raman frequency shift as a function of carrier concentration, $N$. For n-type $\mathrm{Si}$ this is given by:

$10^{3}\left(\frac{\Delta \omega}{\omega_{\mathrm{p}}}\right)=-0.025 X\left(1+0.02 X^{2 / 3}\right)$

where $X=10^{-19} N$ and $\omega_{\mathrm{p}}$ and $\Delta \omega$ are the wave number of the first order Raman band in the absence of strain or disorder effects and the Raman shift from this position, respectively. The carrier concentration calculated from the Raman shift using UV Raman will reflect the average carrier concentration over the probed volume to a depth of $\sim 9 \mathrm{~nm}$. The average carrier concentration of a Sb-implanted strained Si sample annealed at $700{ }^{\circ} \mathrm{C}$ measured using the differential Hall technique is $7.1 \times 10^{20} \mathrm{~cm}^{-3}$ [11]. By way of example, from Eq. 4 this would imply a Raman peak shift of $-1.24 \mathrm{~cm}^{-1}$, and would therefore largely account for the measured peak shift of $-1.32 \mathrm{~cm}^{-1}$ at $700{ }^{\circ} \mathrm{C}$ plotted in Fig. 5. However, at this stage of our investigations we would not rule out a further contribution to this peak shift due to a small change in strain following activation of dopants by heat treatment.

\section{Conclusions}

$\mathrm{Sb}$ is suggested as a possible alternative dopant to As for use in ultrashallow source-drain regions in future CMOS devices. A significant drop in resistivity is measured for $\mathrm{Sb}$ compared to As doping in strained Si. Micro-Raman spectroscopy has shown that the damage induced by low energy ion-implantation is mostly repaired by low thermalbudget heat treatment in the range of $600-800{ }^{\circ} \mathrm{C}$. A correlation has been found between the frequency of the $\mathrm{Si}$ Raman peak and the electron carrier concentration.

Acknowledgements Science Foundation Ireland is gratefully acknowledged for funding this project under the Investigator Programme Grant. The authors would like to thank IQE Silicon Compounds Ltd. for providing the strained silicon substrates used in these experiments. We acknowledge ANKA, HASYLAB and the European Community for funding under Contract RII3-CT-2004_506009 (IASFS). We are grateful to R. Simon of ANKA for assistance in using the Topas beamline and T. Wroblewski and C. Paulmann for their help at HASYLAB beamline F-1.

\section{References}

1. S. Thompson, P. Packan, M. Bohr, Intel Technol. J. Q3'98

2. T. Alzanki, R. Gwilliam, N. Emerson, B.J. Sealy, Appl. Phys. Lett. 85, 1979 (2004)

3. N.S. Bennett, A.J. Smith, C.S. Beer, L. O'Reilly, B. Colombeau, G.D. Dilliway, R. Harper, P.J. McNally, R. Gwilliam, N.E.B. Cowern, B.J. Sealy, Mater. Res. Soc. Symp. Proc. 912, C2.3 (2006)

4. G.D.M. Dilliway, A.J. Smith, J.J. Hamilton, J. Benson, L. Xu, P.J. McNally, G. Cooke, H. Kheyrandish, N.E.B. Cowern, Proc. IIT NIM-B 237, 131 (2005)

5. I. de Wolf, Semicond. Sci. Technol. 11, 139 (1996)

6. B. Pichaud, M. Putero, N. Burle, phys. stat. sol. (a) 171, 251 (1999)

7. J. Macía, E. Martín, A. Pérez-Rodríguez, J. Jiménez, J.R. Morante, B. Aspar, J. Margail, J. Appl. Phys. 82, 3730 (1997)

8. F. Cerdeira, M. Cardona, Phys. Rev. B 5, 1440 (1972)

9. H. Richter, Z.P. Wang, L. Ley, Solid State Commun. 39, 625 (1981)

10. I.H. Campbell, P.M. Fauchet, Solid State Commun. 58, 739 (1986)

11. N.S. Bennett, N.E.B. Cowern, A.J. Smith, R.M. Gwilliam, B.J. Sealy, L. O'Reilly P.J. McNally, G. Cooke, H. Kheyrandish, Appl, Phys. Lett. 89, 182122 (2006) 